\title{
BMJ Open Study protocol for the safety and efficacy of probiotic therapy on days alive and out of hospital in adult ICU patients: the multicentre, randomised, placebo-controlled Restoration Of gut microflora in Critical Illness Trial (ROCIT)
}

Edward Litton (D) , ${ }^{1,2}$ Matthew Anstey, ${ }^{3}$ David Broadhurst, ${ }^{4}$ Andy R Chapman, ${ }^{5}$ Andrew Currie, ${ }^{6}$ Janet Ferrier, ${ }^{2}$ Joel Gummer, ${ }^{6}$ Alisa Higgins, ${ }^{7}$ Jolene Lim, ${ }^{1}$ Laurens Manning, ${ }^{8}$ Erina Myers, ${ }^{3}$ Katrina Orr, ${ }^{9}$ Anne-Marie Palermo, ${ }^{1}$ Andrea Paparini, ${ }^{6,10}$ Susan Pellicano, ${ }^{1}$ Edward Raby, ${ }^{11}$ Anu Rammohan, ${ }^{12}$ Adrian Regli, ${ }^{1,13}$ Bernhard Richter, ${ }^{1,14}$ Sam Salman, ${ }^{8}$ Tobias Strunk, ${ }^{15}$ Sharon Waterson, ${ }^{5}$ Brad Wibrow, ${ }^{3}$ Fiona M Wood ${ }^{8,16}$

To cite: Litton E, Anstey M, Broadhurst D, et al. Study protocol for the safety and efficacy of probiotic therapy on days alive and out of hospital in adult ICU patients: the multicentre, randomised, placebo-controlled Restoration Of gut microflora in Critical Illness Trial (ROCIT). BMJ Open 2020;10:e035930. doi:10.1136/ bmjopen-2019-035930

- Prepublication history and additional material for this paper are available online. To view these files, please visit the journal online (http://dx.doi. org/10.1136/bmjopen-2019035930).

Received 25 November 2019 Revised 06 February 2020 Accepted 06 April 2020

A) Check for updates

(c) Author(s) (or their employer(s)) 2020. Re-use permitted under CC BY-NC. No commercial re-use. See rights and permissions. Published by BMJ.

For numbered affiliations see end of article.

Correspondence to

Dr Edward Litton;

ed.litton@health.wa.gov.au

\section{ABSTRACT}

Introduction The effect of early and sustained administration of daily probiotic therapy on patients admitted to the intensive care unit (ICU) remains uncertain. Methods and analysis The Restoration of gut microflora in Critical IIIness Trial (ROCIT) study is a multicentre, randomised, placebo-controlled, parallel-group, two-sided superiority trial that will enrol 220 patients in five ICUs. Adult patients who are within 48 hours of admission to an ICU and are expected to require intensive care beyond the next calendar day will be randomised in a 1:1 ratio to receive early and sustained Lactobacillus plantarum 299v probiotic therapy in addition to usual care or placebo in addition to usual care. The primary endpoint is days alive and out of hospital to day 60 .

Ethics and dissemination ROCIT has been approved by the South Metropolitan Health Service Human Research Ethics Committee (ref: RGS00000004) and the St John of God Health Care Human Research Ethics Committee (ref: 1183). The trial results will be submitted for publication in a peer-reviewed journal.

Trial registration number Australian and New Zealand Clinical Trials Registry (ANZCTR12617000783325); Preresults.

\section{INTRODUCTION}

Patients admitted to the intensive care unit (ICU) commonly develop dysbiosis, an imbalance in intestinal commensal microflora characterised by a decrease in the diversity of commensal gut bacteria and an overgrowth of pathogenic species that is associated with increased morbidity and mortality. ${ }^{1-5}$
Strengths and limitations of this study

- Early and sustained administration of study drug until the determination of the primary outcome (day $60)$.

- Pragmatic study design including broad eligibility criteria and administration in a usual care setting.

- Sample size calculation informed by consumers and local baseline data.

- Blinded adjudication of outcomes including nosocomial infection.

- A requirement to deliver and assess the delivery of study drug beyond intensive care unit and hospital discharge.

Probiotics are live microorganisms that, when administered in adequate amounts, confer a beneficial effect on the health of the host. ${ }^{6}$ Probiotic therapy may reduce the incidence of surgical-site infections and other postoperative complications in patients undergoing surgery. ${ }^{7}$ In patients admitted to the ICU, probiotic therapy may reduce the risk of nosocomial infections and reduce the hospital length of stay (LOS).$^{8-11}$ A recent metaanalysis of 30 randomised controlled trials (RCTs) concluded that probiotic therapy was associated with a significant reduction in infection, but had no significant effect on mortality. ${ }^{12}$ However, study design heterogeneity and risk of bias have precluded strong 
recommendations for the use of probiotics in current critical care nutrition guidelines. ${ }^{13} 14$ Furthermore, existing RCTs have generally not addressed the attributable risk of nosocomial infection, morbidity and mortality that persists after discharge from an index ICU admission. ${ }^{15}$

Among available probiotic strains, Lactobacillus plantarum $299 \mathrm{v}(\mathrm{Lp} 299 \mathrm{v})$ is a strong candidate therapy to improve outcomes in critically ill patients. Administration results in intestinal colonisation and survival of the probiotic through the entire gastrointestinal tract, regardless of gastric $\mathrm{pH} .{ }^{16-18}$ In otherwise healthy smokers, Lp299v therapy decreases markers of inflammation and oxidative stress. ${ }^{19}$ In a recent landmark trial, Lp299v therapy reduced sepsis and death in rural Indian newborns. ${ }^{20}$ In critically ill patients admitted to the ICU, Lp299v therapy exhibits similar suppression of oropharyngeal colonisation with pathogenic bacteria as chlorhexidine, reduces colonic colonisation with Clostridiodes difficile and attenuates markers of systemic inflammation. ${ }^{21-23}$ The possibility of specific benefit from Lp299v therapy in patients admitted to the ICU is supported by a meta-analysis reporting that although probiotic therapy appears to reduce nosocomial infection in critical illness, a significant benefit is only evident in trials administering Lp299v. ${ }^{12}$ However, recent evidence suggests that probiotic lactobacilli strains can directly cause bacteraemia when administered to patients in ICU and the safety and efficacy of Lp229v in adult patients admitted to the ICU remains uncertain. ${ }^{24}$

The Restoration Of gut microflora in Critical Illness Trial (ROCIT) was designed to assess whether, in adult patients admitted to the ICU, early and sustained daily administration of probiotic therapy using Lp299v, compared with placebo, is associated with an increase in days alive and out of hospital to day $60\left(\mathrm{DAOH}_{60}\right)$.

This report describes the ROCIT protocol and statistical analysis plan.

\section{METHODS AND ANALYSIS \\ Trial design}

ROCIT is a multicentre, placebo-controlled, parallelgroup, two-sided superiority trial that will randomly allocate patients admitted to the ICU in a 1:1 ratio. Participants will receive probiotics in addition to usual care, or placebo in addition to usual care. ROCIT has been designed with reference to the Standard Protocol Items: Recommendations for Interval Trials checklist and is informed by consumer consultation (Consumer and Community Health Research Network, University of Western Australia, WA) ${ }^{25}$ The trial was prospectively registered on the Australian and New Zealand Clinical Trials Registry.

\section{Setting and participants}

ROCIT will enrol a total of 220 participants from five study sites in Western Australia (see the supplementary appendix for the study site list). Eligible patients are those within 48 hours of ICU admission and who are expected to remain in the ICU beyond the next calendar day. ICU admission includes admission to a high-dependency area, defined as an area capable of providing invasive monitoring and a nursing ratio of no greater than 1:2. Patients who will be excluded include those with an absolute contraindication to receiving medication via the enteral route and those with one or more risk factors for treatment-associated adverse effects including recent or

Table 1 Trial eligibility criteria

1. Adult patient within 48 hours of admission to an ICU

Inclusion criteria

2. Expected to require ICU-level care beyond the next calendar day

Exclusion criteria

1. <18years of age

2. Absolute contraindication to receiving medication via the enteral route

3. Known to be receiving probiotic therapy at the time of index hospitalisation

4. Acute pancreatitis as a cause or complication of current admission

5. Immunosuppression (defined as chemotherapy within the preceding 4 weeks or receiving $\geq 1.5 \mathrm{mg} / \mathrm{kg}$ methylprednisolone daily or equivalent)

6. Neutropenia (neutrophil count $\leq 1 \times 10^{9} / \mathrm{L}$ )

7. Prosthetic heart valve or permanent pacemaker

8. Death is deemed to be inevitable as a result of the current acute illness AND either the treating clinician, the patient or the substitute decision-maker, are not committed to full active treatment

9. Enrolment is not considered in the patient's best interest

10. Previously enrolled in ROCIT

11. Unlikely to be residing near or visiting a study centre in 60 days

12. Participating in a competing interventional study

13. Pregnancy

14. Admitted to hospital from a high-level nursing facility or rehabilitation facility

ICU, intensive care unit; ROCIT, Restoration Of gut microflora in Critical Illness Trial. 


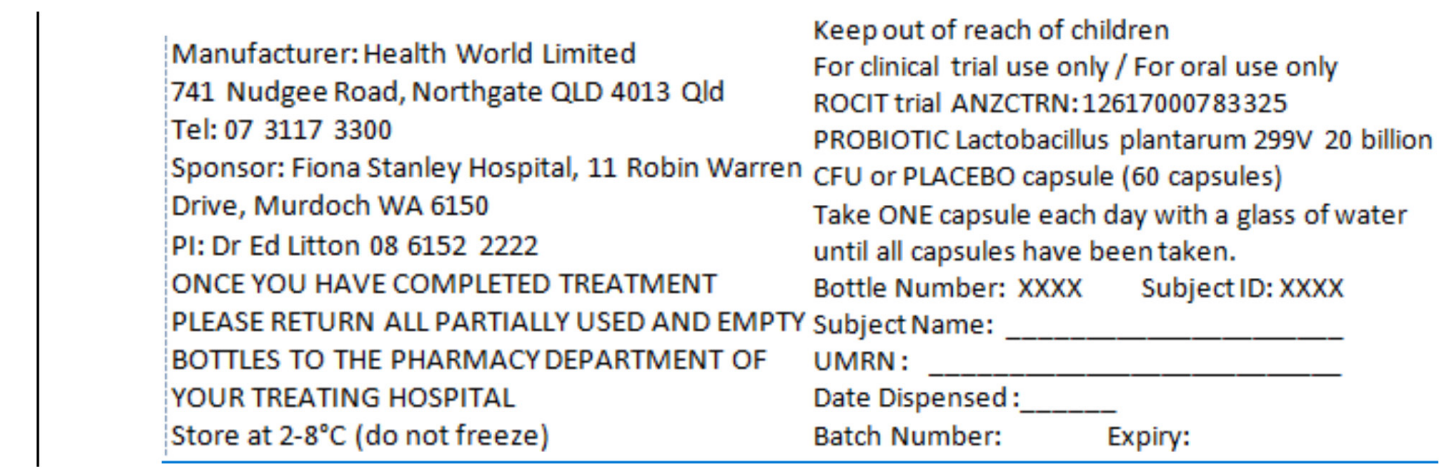

ongoing immunosuppressive therapy. ${ }^{26}$ The complete inclusion and exclusion criteria are provided in table 1. The first patient was enrolled on 28 July 2017 and recruitment to the planned sample size is expected to be completed in early 2020.

\section{Randomisation and blinding}

Eligible participants are identified by members of the study and clinical teams at participating sites. This pragmatic approach, embedded in clinical care, will maximise recruitment. The variable-block randomisation algorithm is stratified by site and has been generated using a web-based randomisation interface by an unblinded pharmacist with no direct involvement in patient care, data collection or analysis. ${ }^{27}$ Allocation concealment is maintained by assigning a unique number to each bottle of study drug (figure 1). The randomisation list is kept by the unblinded pharmacist who is also available for unblinding at the request of the patient or treating team. After trial enrolment, the participant is assigned the next available subject number, corresponding to the unique, consecutively numbered bottle of study drug.

The active study drug and the placebo are prepared in identically packaged capsules and bottles by a certified facility (Health World, Northgate, Queensland, Australia). All treating team members, participants, study staff and outcome adjudicators are blinded to the treatment allocation.

\section{Study treatments}

Immediately after enrolment, a dose of study drug is administered. A single capsule of the study drug is then prescribed daily, beginning the next calendar day. Instructions are provided to continue once daily administration, including after index ICU and hospital discharge, until day 60, (ie, the completion of the 60-capsule bottle). A standard operating procedure is provided to bedside clinical staff for the preparation and administration of study drug and contains instructions for nasogastric tube administration for participants unable to swallow capsules (see online supplementary appendix figure 1). At the time of hospital discharge, participants are provided with a study diary to record daily study drug administration (see online supplementary appendix figure 2). Participants are asked to return the completed diary along with the study drug bottle and any remaining capsules on day 60.

Participants randomly allocated to the active study arm receive a daily capsule with $20 \times 10^{9}$ colony-forming units (CFUs) of Lp299v. Participants randomly allocated to the placebo arm receive an identical-looking capsule of maltodextrin. Independent batch testing of the study drug conducted by members of the study team and provided by the unblinded pharmacist confirmed $>20 \times 10^{9} \mathrm{Lp} 299 \mathrm{v}$ CFU and unrecordable Lp299v CFU in the active and placebo capsules, respectively.

The study drug is transported under controlled and recorded refrigerated conditions from the manufacturer to study sites and stored under refrigerated and monitored conditions during the hospital stay. A cool bag is provided to patients for the transport of the study drug on hospital discharge and patients are advised to refrigerate the study drug as soon as they arrive home. A clinical trial notification for ROCIT has been lodged with the Australian Government Therapeutic Goods Administration (ref: CT-2017-CTN-03 603-1).

\section{Concomitant therapies}

Participants are requested to refrain from initiating any probiotic treatment other than the study treatment during the 60 days of study participation. Probiotics are not on the hospital formulary of any of the five study sites participating ROCIT. All other care is at the discretion of the treating teams.

\section{Discontinuation}

Study drug may be discontinued at the request of the participant or treating clinician at any stage if the participant or treating clinician suspects an adverse reaction or that continued participation is not in the best interest of the participant. A suspected or confirmed severe adverse drug reaction will result in immediate and permanent discontinuation of the study medication. Study drug will also be discontinued permanently if $L$. plantarum is grown from a sterile site or is the predominant growth from a non-sterile site.

\section{Outcomes}

The flow of participants in the study will be reported according to Consolidated Standards of Reporting Trials' 


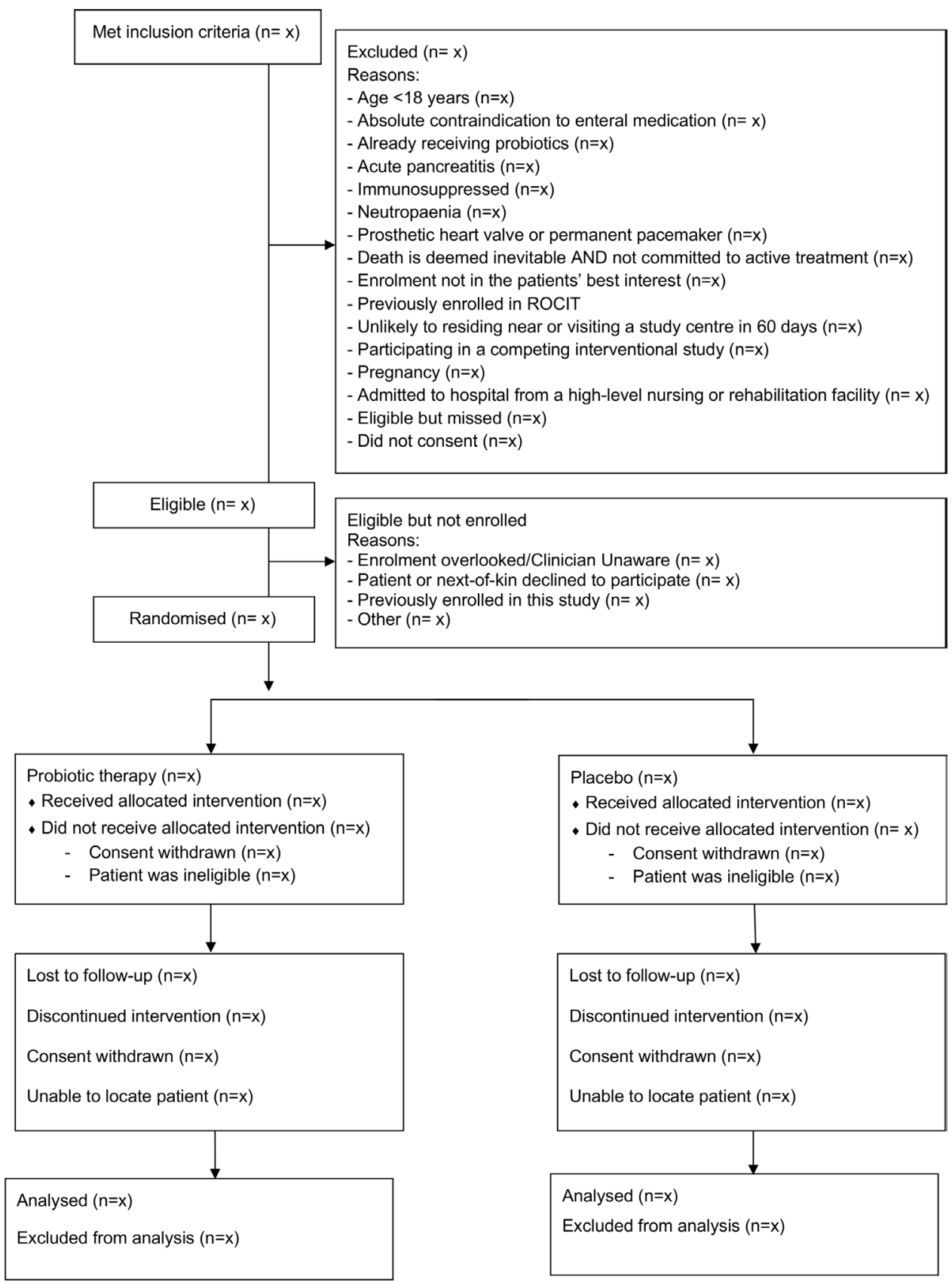

Figure 2 Proposed reporting of the flow of trial participants. ROCIT, Restoration Of gut microflora in Critical Illness Trial.

criteria (figure 2). ${ }^{28}$ The primary outcome is $\mathrm{DAOH}_{60}$. $\mathrm{DAOH}$ is a validated measure that includes death, LOS in hospital, need for ongoing rehabilitation and the occurrence and duration of hospital readmission. ${ }^{29-32}$ Days spent in a rehabilitation facility or high-level nursing facility to day 60 are considered as days in the hospital. Participants who die prior to day 60 will be recorded as having zero $\mathrm{DAOH}_{60}{ }^{33}$

Secondary endpoints include the occurrence of specified nosocomial infections (hospital-acquired pneumonia, ventilator-associated pneumonia, C. difficileassociated diarrhoea, surgical-site infection, urinary tract infection and blood stream infection) defined according to Centre for Disease Control criteria (see online supplementary appendix table 1$) \cdot{ }^{34}$ Screening for nosocomial infection will occur by identifying each episode of initiation or change of antibiotic to day 60 and will then be assessed independently by two blinded infectious diseases specialist clinicians by review of the medical records. Any disagreement will be resolved by consensus. Other secondary endpoints include antibiotic-free days to day 60, ICU and hospital LOS, and ICU, hospital and 60-day mortality. Quality of life will be assessed using the fivelevel EuroQol five-dimension questionnaire at day 60, administered via telephone by blinded research staff at each study site (table 2).

\section{Data collection and management}

Trained research coordinators will collect data at each site using a study-specific case report form. Study data 
Table 2 Study data to be collected

\begin{tabular}{|c|c|}
\hline Time point & Study data \\
\hline Screening & $\begin{array}{l}\text { Date of screening } \\
\text { Inclusion and exclusion criteria } \\
\text { Reason, if not enrolled } \\
\text { Study number and patient initials for enrolled participants }\end{array}$ \\
\hline Baseline & $\begin{array}{l}\text { Date and time of randomisation } \\
\text { Date and time of ICU admission } \\
\text { Demographic data } \\
\text { - ICU admission source and category } \\
\text { Nutrition, acid-suppressive therapy and antibiotics } \\
\text { - Admission APACHE II score, diagnostic code and comorbidities } \\
\text { - SOFA Score and components } \\
\text { - Mechanical ventilation } \\
\text { Vasoactive medication } \\
\text { Renal replacement therapy }\end{array}$ \\
\hline Outcome (day 60) & $\begin{array}{l}\text { Hospital length of stay } \\
\text { Nosocomial infection (hospital-acquired pneumonia, ventilator-associated pneumonia, Clostridium } \\
\text { difficile-associated diarrhoea, surgical-site infection, urinary tract infection and blood stream infection) } \\
\text { ICU length of stay } \\
\text { ICU mortality } \\
\text { Hospital mortality } \\
\text { - EQ-5D-5L }\end{array}$ \\
\hline Adverse events & Description, timing, causality and resolution of adverse events from randomisation to day 60 \\
\hline
\end{tabular}

*The prespecified nosocomial infections will be identified according to the Centre for Disease Control definitions and are provided in the online supplementary appendix.

APACHE, Acute Physiology And Chronic Health Evaluation; EQ-5D-5L, five-level EuroQol five-dimension questionnaire; HDA, highdependency area; ICU, intensive care unit; SOFA, Sequential Organ Failure Score.

are entered into a Research Electronic Data Capture (REDCap) database, a secure, web-based software platform. ${ }^{35}$ Assessment of the primary outcome will include direct phone contact with participants on or shortly after day 60 where participants are not known to be hospitalised or have died. Details of the occurrence and duration of hospital readmissions will be collected during this phone call and cross-checked against hospital medical records and, if required, general practitioner records. To ensure the accuracy and completeness of data, there will be prespecified automatic checks and on-site data monitoring by the project manager, including $100 \%$ source data verification for the primary endpoint. Screening, baseline, daily, outcome, adverse event and protocol deviation data are provided in table 2. The plans for collecting and storing biological specimens for analysis in ancillary studies are provided in the supplementary appendix.

\section{Sample size and power}

A difference of 4 days in $\mathrm{DAOH}_{60}$ is considered meaningful by a specially convened forum of consumers including ICU survivors and next-of-kin (Consumer and
Community Health Research Network, University of Western Australia, WA). Baseline $\mathrm{DAOH}_{60}$ has been calculated using contemporary data from participating hospitals. From these data, a baseline of $37 \mathrm{DAOH}_{60}$, an $\mathrm{SD}$ of 9 and a two-tailed $\alpha=0.05$, a trial of 162 participants has $80 \%$ power to detect a difference in $\mathrm{DAOH}_{60}$ of 4 days. After inflation for non-normal distribution (20\%), withdrawn consent (5\%) and loss to follow-up (5\%), the final sample size is 220 participants.

\section{Statistical analysis plan}

The primary analysis will be the intention-to-treat population, defined as all eligible and randomised study participants, except for those who do not consent to use of the data necessary to determine the primary outcome. There will be no imputation for missing data. Normally distributed data will be presented as mean (SD) and nonnormally distributed data as median (IQR). Comparisons will be performed using Fisher's exact test for categorical data and Student's t test or the Wilcoxon rank-sum test for normally and non-normally distributed data, respectively. The primary outcome $\left(\mathrm{DAOH}_{60}\right)$, will be analysed 
using the Wilcoxon rank-sum test with results presented as a comparison of medians (IQR). A two-sided $p$ value of $<0.05$ will be considered statistically significant. Heterogeneity between prespecified subgroups, identified at baseline, will be assessed by fitting an interaction term between treatment and subgroup.

The three subgroup pairs will be: patients with sepsis versus those without sepsis; emergency versus elective ICU admissions; and surgical versus medical admissions. A perprotocol analysis will be conducted including all participants with reported adherence to the study medication for $>80 \%$ of their total study duration. Planned substudies include longitudinal evaluation of faecal microbiome and blood metabolome, and if there is a statistically significant difference in the primary outcome, then an economic evaluation of the cost-effectiveness of the intervention will be there. All analyses will be conducted using STATA/SE V.13.

\section{Patient and public involvement}

The primary outcome was chosen on the basis of published evidence of the importance placed by patients on days spent at home. ${ }^{36}$ Consideration of additional outcome measures was made in conjunction with an ICU consumer forum convened from the Consumer and Community Health Research Network (University of Western Australia, WA). Study participants are offered the opportunity to have the published study results supplied to them directly and to be unblinded after the final determination of all study outcome measures. The published manuscript of the primary outcome will be made available to the Consumer and Community Health Research Network for dissemination among stakeholders.

\section{Data monitoring committee}

The data monitoring committee (DMC) has expertise in critical care, infectious diseases and trial design but is not otherwise involved in the care of study participants and is independent of competing interests. The members are Nolan McDonnell (BHB, MBChB, FANZCA, MClinRes), Claire Italiano (MBBS, FRACP, MPHTM) and Ravi Sonowane (MBBS, FCICM, MPH). The DMC has reviewed and approved the study protocol and will review all serious adverse events as they occur. The ROCIT management committee will inform the DMC of any accumulating external evidence of relevance to the ongoing conduct of the study as soon as practicable. No interim analyses are planned but the DMC will reserve the right to conduct an interim analysis or advise suspension or termination of ongoing enrolment to the study.

\section{Adverse events}

Events that are a part of the natural history of the primary disease process or expected complication of critical illness will not be reported as serious adverse events. ${ }^{37}$ All adverse events considered to be potentially causally related to the trial, and all serious adverse events will be reported (online supplementary appendix table 2 ).

\section{ETHICS AND DISSEMINATION}

\section{Ethics approval}

ROCIT has been approved by the South Metropolitan Health Service Human Research Ethics Committee (ref: RGS00000004) and the St John of God Health Care Human Research Ethics Committee (ref: 1183). The approved consent pathways included prospective participant consent for study-eligible patients with capacity and prospective person responsible acknowledgement with deferred consent for patients who lacked capacity. Protocol modifications will be submitted to the Human Research Ethics Committee review prior to dissemination and initiation at trial sites. A copy of the consent form is provided in the supplementary appendix.

\section{Dissemination}

The study results will be submitted for publication in a peer-reviewed journal. Study data and statistical code can be accessed by contacting the corresponding author. Requests for access will be reviewed by the named authors on a case-by-case basis.

\section{Author affiliations}

${ }^{1}$ Intensive Care Unit, Fiona Stanley Hospital, Murdoch, Western Australia, Australia ${ }^{2}$ Intensive Care Unit, St John of God Hospital, Subiaco, Western Australia, Australia ${ }^{3}$ Intensive Care Unit, Sir Charles Gairdner Hospital, Nedlands, Western Australia, Australia

${ }^{4}$ School of Science, Edith Cowan University, Joondalup, Western Australia, Australia ${ }^{5}$ Intensive Care Unit, Royal Perth Hospital, Perth, Western Australia, Australia

${ }^{6}$ Murdoch University, Murdoch, Western Australia, Australia

${ }^{7}$ Australian and New Zealand Intensive Care Research Centre, Monash University, Clayton, Victoria, Australia

${ }^{8}$ University of Western Australia, Perth, Western Australia, Australia

${ }^{9}$ Pharmacy, Fiona Stanley Hospital, Murdoch, Western Australia, Australia

${ }^{10}$ Econumerics Pty Ltd consultants, Perth, Western Australia, Australia

${ }^{11}$ Department of Infectious Diseases, Fiona Stanley Hospital, Murdoch, Western Australia, Australia

${ }^{12}$ Department of Economics, University of Western Australia, Crawley, Western Australia, Australia

${ }^{13}$ Intensive Care Unit, St John of God Hospital, Murdoch, Western Australia, Australia

${ }^{14}$ Division of Cardiology, Medical University of Vienna, Wien, Wien, Austria

${ }^{15}$ Neonatal Directorate, King Edward Memorial Hospital for Women Perth, Subiaco, Western Australia, Australia

${ }^{16}$ Burns Department, Fiona Stanley Hospital, Murdoch, Western Australia, Australia

Correction notice This article has been corrected since it was published. Name for the Andrea Paparini has been corrected.

\section{Twitter Edward Litton @ed_litton}

Acknowledgements The authors would like to acknowledge and thank all participants of the ROCIT study and the consumers from the Consumer and Community Health Research Network (University of Western Australia, WA) for their contribution to the study design.

Contributors EL has made a substantial contribution to the design of the work and drafting the work and has given final approval for the work and agrees to be accountable for all aspects of the work. MA, DB, ACh, ACu, JF, JG, AH, JL, LM, EM, KO, A-MP, AP, SP, ER, ARa, ARe, BR, SS, TS, SW, BW, FW has made a substantial contribution to the design of the work and revising the work and has given final approval for the work and agrees to be accountable for all aspects of the work.

Funding ROCIT is funded by grants from the Department of Health, Government of Western Australia Research Translation Projects, the St John of God Hospital Foundation and the Fiona Wood Foundation. Study drug was supplied by Health World. The funding bodies and Health World had no input into the design or conduct of the trial and will have no input into the analysis or reporting of the trial 
results. The study sponsor is the Fiona Stanley Fremantle Hospital Group, South Metropolitan Health Service, Western Australia.

Competing interests None declared.

Patient and public involvement Patients and/or the public were involved in the design, or conduct, or reporting, or dissemination plans of this research. Refer to the Methods section for further details.

Patient consent for publication Not required.

Provenance and peer review Not commissioned; externally peer reviewed.

Open access This is an open access article distributed in accordance with the Creative Commons Attribution Non Commercial (CC BY-NC 4.0) license, which permits others to distribute, remix, adapt, build upon this work non-commercially, and license their derivative works on different terms, provided the original work is properly cited, appropriate credit is given, any changes made indicated, and the use is non-commercial. See: http://creativecommons.org/licenses/by-nc/4.0/.

ORCID iD

Edward Litton http://orcid.org/0000-0002-5125-6829

\section{REFERENCES}

1 Petersen C, Round JL. Defining dysbiosis and its influence on host immunity and disease. Cell Microbiol 2014;16:1024-33.

$2 \mathrm{KM} \mathrm{H}$, Kalgudi S, Corbett JM, et al. Gut microbiota in surgical and critically ill patients. Anaesthesia and Intensive Care 2020.

3 Shimizu K, Ogura H, Goto M, et al. Altered gut flora and environment in patients with severe SIRS. J Trauma 2006;60:126-33.

4 Ojima M, Motooka D, Shimizu K, et al. Metagenomic analysis reveals dynamic changes of whole gut microbiota in the acute phase of intensive care unit patients. Dig Dis Sci 2016;61:1628-34.

5 McDonald D, Ackermann G, Khailova L, et al. Extreme dysbiosis of the microbiome in critical illness. mSphere 2016;1. doi:10.1128/ mSphere.00199-16. [Epub ahead of print: 31 Aug 2016].

6 Food and Agriculture Organization of the United Nations and World Health Organization. Report of a joint FAO/WHO expert consultation on evaluation of health and nutritional properties of probiotics in food including powder milk with live lactic acid bacteria. 2001. Cordoba, Argentina: FAO/WHO, 2001.

7 Skonieczna-Żydecka K, Kaczmarczyk M, Łoniewski I, et al. A systematic review, meta-analysis, and meta-regression evaluating the efficacy and mechanisms of action of probiotics and synbiotics in the prevention of surgical site infections and surgery-related complications. J Clin Med 2018;7:556.

8 Kotzampassi K, Giamarellos-Bourboulis EJ, Voudouris A, et al. Benefits of a synbiotic formula (synbiotic 2000Forte) in critically ill trauma patients: early results of a randomized controlled trial. World J Surg 2006;30:1848-55.

9 Barraud D, Blard C, Hein F, et al. Probiotics in the critically ill patient: a double blind, randomized, placebo-controlled trial. Intensive Care Med 2010;36:1540-7.

10 Petrof EO, Dhaliwal R, Manzanares W, et al. Probiotics in the critically ill: a systematic review of the randomized trial evidence. Crit Care Med 2012;40:3290-302.

11 Mahmoodpoor A, Hamishehkar H, Asghari R, et al. Effect of a probiotic preparation on ventilator-associated pneumonia in critically ill patients admitted to the intensive care unit: a prospective doubleblind randomized controlled trial. Nutrition in Clinical Practice 2019;34:156-62.

12 Manzanares W, Lemieux M, Langlois PL, et al. Probiotic and synbiotic therapy in critical illness: a systematic review and metaanalysis. Critical Care 2016;20:262.

13 McClave SA, Taylor BE, Martindale RG, et al. Guidelines for the provision and assessment of nutrition support therapy in the adult critically ill patient: Society of critical care medicine (SCCM) and American Society for parenteral and enteral nutrition (A.S.P.E.N.). JPEN J Parenter Enteral Nutr 2016;40:159-211.

14 Dhaliwal R, Cahill N, Lemieux M, et al. The Canadian critical care nutrition guidelines in 2013: an update on current recommendations and implementation strategies. Nutr Clin Pract 2014;29:29-43.
15 Ho KM, Kalgudi S, Corbett J-M, et al. Gut microbiota in surgical and critically ill patients. Anaesth Intensive Care 2020;310057X:20903732.

16 Goossens D, Jonkers D, Russel M, et al. Survival of the probiotic, L. plantarum 299v and its effects on the faecal bacterial flora, with and without gastric acid inhibition. Dig Liver Dis 2005;37:44-50.

17 Johansson ML, Molin G, Jeppsson B, et al. Administration of different Lactobacillus strains in fermented oatmeal soup: in vivo colonization of human intestinal mucosa and effect on the Indigenous flora. Appl Environ Microbiol 1993;59:15-20.

18 Klarin B, Johansson M-L, Molin G, et al. Adhesion of the probiotic bacterium Lactobacillus plantarum 299v onto the gut mucosa in critically ill patients: a randomised open trial. Crit Care 2005;9:R285-93.

19 Naruszewicz M, Johansson M-L, Zapolska-Downar D, et al. Effect of Lactobacillus plantarum $299 \mathrm{v}$ on cardiovascular disease risk factors in smokers. Am J Clin Nutr 2002;76:1249-55.

20 Panigrahi P, Parida S, Nanda NC, et al. A randomized synbiotic trial to prevent sepsis among infants in rural India. Nature 2017;548:407-12.

21 Klarin B, Wullt M, Palmquist I, et al. Lactobacillus plantarum 299v reduces colonisation of Clostridium difficile in critically ill patients treated with antibiotics. Acta Anaesthesiol Scand 2008;52:1096-102.

22 Klarin B, Adolfsson A, Torstensson A, et al. Can probiotics be an alternative to chlorhexidine for oral care in the mechanically ventilated patient? A multicentre, prospective, randomised controlled open trial. Critical Care 2018;22:272.

23 McNaught CE, Woodcock NP, Anderson ADG, et al. A prospective randomised trial of probiotics in critically ill patients. Clin Nutr 2005;24:211-9.

24 Yelin I, Flett KB, Merakou C, et al. Genomic and epidemiological evidence of bacterial transmission from probiotic capsule to blood in ICU patients. Nat Med 2019;25:1728-32.

25 Chan A-W, Tetzlaff JM, Gøtzsche PC, et al. Spirit 2013 explanation and elaboration: guidance for protocols of clinical trials. BMJ 2013;346:e7586.

26 Kochan P, Chmielarczyk A, Szymaniak L, et al. Lactobacillus rhamnosus administration causes sepsis in a cardiosurgical patient-is the time right to revise probiotic safety guidelines? Clin Microbiol Infect 2011;17:1589-92.

27 sealedenvelope.com. Clerkenwell workshops, London EC1R OAT, UK, 2019 [Accessed 11 Oct 2019].

28 Schulz KF, Altman DG, Moher D, et al. Consort 2010 statement: updated guidelines for reporting parallel group randomised trials. BMJ 2010;340:c332.

29 Ariti CA, Cleland JGF, Pocock SJ, et al. Days alive and out of hospital and the patient journey in patients with heart failure: insights from the candesartan in heart failure: assessment of reduction in mortality and morbidity (CHARM) program. Am Heart $J$ 2011;162:900-6.

30 Myles PS, Shulman MA, Heritier S, et al. Validation of days at home as an outcome measure after surgery: a prospective cohort study in Australia. BMJ Open 2017;7:e015828.

31 Bell M, Eriksson LI, Svensson T, et al. Days at home after surgery: an integrated and efficient outcome measure for clinical trials and quality assurance. EClinicalMedicine 2019;11:18-26.

32 Jerath A, Austin PC, Wijeysundera DN. Days alive and out of hospital: validation of a patient-centered outcome for perioperative medicine. Anesthesiology 2019;131:84-93.

33 Burns KEA, Jacob SK, Aguirre V, et al. Stakeholder engagement in trial design: survey of visitors to critically ill patients regarding preferences for outcomes and treatment options during weaning from mechanical ventilation. Ann Am Thorac Soc 2016;13:1962-8.

34 CDC. Chapter 17: CDC/NHSN Surveillance Definitions for Specific Types of Infections. In: National healthcare safety network (NHSN) patient safety component manual, 2019.

35 Harris PA, Taylor R, Thielke R, et al. Research electronic data capture (REDCap)--a metadata-driven methodology and workflow process for providing translational research informatics support. J Biomed Inform 2009;42:377-81.

36 Groff AC, Colla CH, Lee TH. Days spent at home - a patient-centered goal and outcome. N Engl J Med 2016;375:1610-2.

37 Cook D, Lauzier F, Rocha MG, et al. Serious adverse events in academic critical care research. CMAJ 2008;178:1181-4. 